\title{
THE UNIVERSAL SEMILATTICE COMPACTIFICATION OF A SEMIGROUP
}

\section{H. R. EBRAHIMI VISHKI and M. A. POURABDOLLAH}

(Received 6 May 1997 and in revised form 10 January 1998)

\begin{abstract}
The universal abelian, band, and semilattice compactifications of a semitopological semigroup are characterized in terms of three function algebras. Some relationships among these function algebras and some well-known ones, from the universal compactification point of view, are also discussed.
\end{abstract}

Keywords and phrases. Semitopological semigroup, (universal) semigroup compactification, weakly (strongly) almost periodic function, distal function, semilattice.

1991 Mathematics Subject Classification. 22A20, 43A60, 22A26, 54H15.

1. Introduction. The notion of semigroup compactification has been produced in several principal ways, in whose main approach the Gelfand-Naimark theory of commutative $C^{*}$-algebras is employed. In fact, the spectrum of every $m$-admissible algebra of functions is a semigroup compactification. Moreover, some of these compactifications enjoy a universal property $P$. For instance, De Leeuw and Glicksberg in their influential paper [2], characterized the universal property of (weakly) almost periodic compactification. The existence of the universal $P$-compactification (using the subdirect product methods) for a broad variety of properties $P$, is guaranteed by Junghenn and Pandian [7]. The construction of some of the better known universal $P$-compactifications in terms of $m$-admissible algebras of functions are collected in Berglund et al. [1], which is our ground reference. The universal right simple, left simple, and group compactifications are characterized in terms of some types of distal functions [6]. In two recent papers [9, 10], Pandian has examined the universal mapping property of generalized distal, and quasiminimal distal functions. Also, in an earlier paper [3], we have characterized the universal nilpotent group compactification. The present paper deals with the construction of three $m$-admissible algebras $A B, B D$, and $S L$, which characterize the universal abelian, band, and semilattice compactifications of a semitopological semigroup.

2. Preliminaries. For background and notations we follow Berglund et al. [1] as much as possible. In what follows, $S$ is a semitopological semigroup unless otherwise stipulated. A (semigroup) compactification of $S$ is a pair $(\psi, X)$, where $X$ is compact, Hausdorff, right topological semigroup and $\psi: S \longrightarrow X$ is a continuous homomorphism with dense image such that, for all $s \in S$, the mapping $x \longmapsto \psi(s) x: X \longrightarrow X$ is continuous.

The $C^{*}$-algebra of all continuous bounded complex-valued functions on a topological space $Y$ is denoted by $C(Y)$. For $C(S)$ left and right translations, $L_{s}$ and $R_{t}$, are 
defined for all $s, t \in S$ by $\left(L_{s} f\right)(t)=f(s t)=\left(R_{t} f\right)(s), f \in C(S)$. A left translation invariant $C^{*}$-subalgebra $F$ of $C(S)$ (i.e., $L_{s} f \in F$ for all $s \in S$ and $f \in F$ ), containing the constant functions, is called $m$-admissible if the function $s \longmapsto\left(T_{\mu} f\right)(s)=\mu\left(L_{s} f\right)$ is in $F$ for all $f \in F$ and $\mu \in S^{F}$ ( = the spectrum of $F$ ). If so, $S^{F}$ under the multiplication $\mu \nu=\mu \circ T_{v}\left(\mu, v \in S^{F}\right)$, furnished with the Gelfand topology, makes $\left(\varepsilon, S^{F}\right)$ a compactification (called the $F$-compactification) of $S$, where $\varepsilon: S \longrightarrow S^{F}$ is the evaluation mapping. Conversely, if $(\psi, X)$ is a compactification of $S$, then $\psi^{*}(C(X))$ is an $m$-admissible subalgebra of $C(S)$, where $\psi^{*}$ is the dual mapping of $\psi$, and this correspondence between compactifications of $S$ and $m$-admissible subalgebras of $C(S)$ is one-to-one (see [1, Thm. 3.1.7]).

A compactification $(\psi, X)$ of $S$, possessing a certain property $P$, is called the universal $P$-compactification if for any other compactification $(\varphi, Z)$, having the property $P$, there exists a homomorphism $\pi:(\psi, X) \longrightarrow(\varphi, Z)$, where $\pi$ is a continuous mapping from $X$ into $Y$ with $\pi \circ \psi=\varphi$, or equivalently, $\varphi^{*}(C(Z)) \subseteq \psi^{*}(C(X)$ ) (see [1, Thm. 3.1.9]).

Some of the usual $m$-admissible subalgebras of $C(S)$, that are needed in the sequel, are the left multiplicatively continuous, weakly almost periodic, almost periodic, strongly almost periodic, distal, minimal distal, and strongly distal functions on $S$. These are denoted by $L M C, W A P, A P, S A P, D, M D$ and $S D$, respectively. We also write $G P$ for $M D \cap S D, L Z$ for $\{f \in C(S): f(s t)=f(s)$ for all $s, t \in S\}$, and $R Z$ for $\{f \in C(S): f(s t)=f(t)$ for all $s, t \in S\}$. Here, and also for other emerging spaces, when there is no risk of confusion, we have suppressed the letter $S$ from the notation. For ease of reference, we mention the next proposition which describes the universal mapping properties of these $m$-admissible algebras.

Proposition 2.1. See [1, Chap. 4] and [6, Thm. 3.4]. The LMC, WAP, AP, SAP, D, MD, $S D, G P, L Z$, and RZ-compactifications are universal with respect to the properties of being a (right topological) semigroup, a semitoplogical semigroup, a topological semigroup, a topological group, an inflation of a rectangular group, a left simple semigroup, a right simple semigroup, a group, a left zero semigroup, and a right zero semigroup, respectively.

3. The main results. To follow the main objective, we examine the properties of $A B$ and $B D$, where

$$
A B=\{f \in W A P: f(s t)=f(t s), \text { and } f(s t u)=f(s u t) \text { for all } s, t, u \in S\}
$$

and $B D$ consists of those $f \in L M C$ such that

$$
\begin{aligned}
& \lim _{\alpha}\left(\lim _{\alpha} R_{s_{\alpha}} f\right)\left(s_{\alpha}\right)=\lim _{\alpha} f\left(s_{\alpha}\right) ; \\
& \lim _{\alpha}\left(\lim _{\alpha} R_{s_{\alpha}}\left(\lim _{\alpha} R_{t_{\alpha}} f\right)\right)\left(s_{\alpha}\right)=\lim _{\alpha}\left(\lim _{\alpha} R_{t_{\alpha}} f\right)\left(s_{\alpha}\right) ; \\
& \lim _{\alpha} R_{s_{\alpha}}\left(\lim _{\alpha} R_{s_{\alpha}} f\right)=\lim _{\alpha} R_{s_{\alpha}} f ; \\
& \lim _{\alpha} R_{s_{\alpha}}\left(\lim _{\alpha} R_{s_{\alpha}}\left(\lim _{\alpha} R_{t_{\alpha}} f\right)\right)=\lim _{\alpha} R_{s_{\alpha}}\left(\lim _{\alpha} R_{t_{\alpha}} f\right)
\end{aligned}
$$

for all nets $\left\{s_{\alpha}\right\}$ and $\left\{t_{\alpha}\right\}$ in $S$ for which the relevant pointwise limits exist. 
Also, we write $S L$ for $A B \cap B D$. The next lemma, which requires a routine proof, characterizes $A B$ and $B D$ in terms of the elements of $S^{W A P}$ and $S^{L M C}$, respectively.

LEMMA 3.1. (i) A function $f \in W A P$ is in $A B$ if and only if $\mu \nu(f)=v \mu(f)$ and $T_{\mu \nu} f=T_{\nu \mu} f$ for all $\mu, v \in S^{W A P}$.

(ii) A function $f \in L M C$ is in BD if and only if $\mu^{2}(f)=\mu(f), \mu^{2} v(f)=\mu \nu(f), T_{\mu^{2}} f=$ $T_{\mu} f$, and $T_{\mu^{2} v} f=T_{\mu \nu} f$ for all $\mu, \nu \in S^{L M C}$.

The following theorem states the main properties of $A B, B D$, and $S L$.

THEOREM 3.2. $A B, B D$, and $S L$ are those $m$-admissible subalgebras of $C(S)$, whose corresponding compactifications of $S$ are universal with respect to the properties of being an abelian semigroup, a band, and a semilattice, respectively.

Proof. It is enough to prove the conclusion for $A B$ and $B D$. Using Lemma 3.1, the $m$-admissibility of $A B$ and $B D$ can be easily demonstrated, and also it follows that $S^{A B}$ and $S^{B D}$ are abelian and a band, respectively. Let $(\psi, X)$ be an abelian compactification of $S$, then $C(X)=A B(X)$ and so $\psi^{*}(C(X))=\psi^{*}(A B(X)) \subseteq A B(S)$, where the latter inclusion can be easily verified. Thus, $\left(\varepsilon, S^{A B}\right)$ is the universal abelian compactification of $S$. Similarly, to see that $\left(\varepsilon, S^{B D}\right)$ is universal with respect to the property of being a band, it is enough to show that for any other band compactification $(\varphi, Z)$ of $S, \varphi^{*}(C(Z)) \subseteq B D(S)$. For this, let $\pi:\left(\varepsilon, S^{L M C}\right) \longrightarrow(\varphi, Z)$ be the canonical homomorphism whose existence is guaranteed by the universal property of $\left(\varepsilon, S^{L M C}\right)$. If $g \in C(Z)$, then $\varphi^{*}(g) \in L M C(S)$ and for all $\mu \in S^{L M C}, \mu^{2}\left(\varphi^{*}(g)\right)=g\left(\pi(\mu)^{2}\right)=$ $g(\pi(\mu))=\mu\left(\varphi^{*}(g)\right)$. A similar argument shows that, for each $v \in S^{L M C}, \mu^{2} v\left(\varphi^{*}(g)\right)=$ $\mu \nu\left(\varphi^{*}(g)\right), T_{\mu^{2}} \varphi^{*}(g)=T_{\mu} \varphi^{*}(g)$, and $T_{\mu^{2} \nu} \varphi^{*}(g)=T_{\mu \nu} \varphi^{*}(g)$. Now, Lemma 3.1 shows that $\varphi^{*}(g) \in B D(S)$, as required.

It is trivial that $B D \subseteq B D_{c}$ (with the equality holding in the compact case), where

$$
\begin{aligned}
B D_{c}=\left\{f \in C(S): f\left(s^{2}\right)=f(s), f\left(s^{2} t\right)\right. & =f(s t)=f\left(s t^{2}\right), \\
& \text { and } \left.f\left(s t^{2} u\right)=f(s t u) \text { for all } s, t, u \in S\right\} .
\end{aligned}
$$

The joint continuity of the multiplication of $S^{A P}$ implies that $B D \cap A P=B D_{\mathcal{C}} \cap A P$. Furthermore, $S^{S L}$ is a compact semitopological semilattice, so by Lawson's (joint continuity) theorem [8], $S L \subseteq A P$. Thus, $S L=A P \cap B D_{c} \cap A B$; more precisely:

Proposition 3.3. $S L=\left\{f \in A P: f\left(s^{2}\right)=f(s), f\left(s^{2} t\right)=f(s t)=f(t s)\right.$, and $f\left(s t^{2} u\right)=f(s t u)=f(s u t)$, for all $\left.s, t, u \in S\right\}$.

The universal properties of $\left(\varepsilon, S^{B D}\right)$ and $\left(\varepsilon, S^{D}\right)$ imply that $\left(\varepsilon, S^{B D \cap D}\right)$ is universal with respect to the property of being a rectangular band [1, Exercise 1.1.48]. Furthermore, since every such rectangular band is a topological semigroup, $B D \cap D \subseteq$ $A P$ which implies that $B D \cap D=B D_{c} \cap D \cap A P$. On the other hand, an adaptation of Junghenn's ideas in the proof of Proposition 3.10 of [6] implies that $B D \cap D=\langle L Z \cup R Z\rangle$ $=L Z \otimes R Z$, where $\langle L Z \cup R Z\rangle$ is the $C^{*}$-subalgebra of $C(S)$ generated by $L Z \cup R Z$ and $L Z \otimes R Z$ is the topological tensor product of $L Z$ and $R Z$; i.e., the completion in the least cross norm of the algebraic tensor product. 
As a consequence of the universal properties of $\left(\varepsilon, S^{G P}\right)$ and $\left(\varepsilon, S^{A B}\right)$, it is trivial that $\left(\varepsilon, S^{A B \cap G P}\right)$ is the universal abelian group compactification of $S$. Some other facts about $A B \cap G P$ are collected in the next result. Also, see [3].

Proposition 3.4. (i) $A B \cap M D=A B \cap G P=A B \cap S D=\{f \in S A P: f(s t u)=f($ sut $)$, for all $s, t, u \in S\}$.

(ii) $A B \cap G P$ is the closed linear span of the set of all continuous characters of $S$.

Proof. The facts that $S^{A B \cap M D}$ and $S^{A B \cap S D}$ are abelian groups and that $\left(\varepsilon, S^{A B \cap G P}\right)$ is universal with respect to the property of being an abelian group imply that $A B \cap M D=$ $A B \cap G P=A B \cap S D \subseteq S A P$, where the latter containment is obtained from the Ellis' (joint continuity) theorem [4]. Furthermore, the other condition in the definition of $A B$, i.e., $f(s t)=f(t s)$ is automatically deduced from $f(s t u)=f(s u t)$ and the fact that $f \in S A P$. The observation that the dual mapping of $\varepsilon$ from $C\left(S^{A B \cap G P}\right)$ onto $A B \cap G P$ establishes a one-to-one correspondence between the continuous characters of $S^{A B \cap G P}$ and those of $S$ and using the Peter-Weyl theorem, [5, Thm. 22.17], for $C\left(S^{A B \cap G P}\right)$ imply that $A B \cap G P$ is the closed linear span of the continuous characters of $S$.

\section{EXAMPLES AND REMARKS 3.5.}

(i) For all right zero and left zero semigroups, it is simple to verify that $A B=\mathbb{C}$ (i.e., consists of the constant functions only) and that $B D=C(S)$. Also, for all groups $B D=\mathbb{C}$.

(ii) Consider the discrete semigroup $S=\{a, b, c, d\}$, with multiplication given by: $a$ as a left identity, $b$ and $c$ be as left zeros, and $d s=c$ for all $s \in S$ (see [1, 1.1.7]). A direct computation shows that $A B=\{f \in C(S): f(b)=f(c)=f(d)\}$ and $B D=\{f \in$ $C(S): f(c)=f(d)\}$.

(iii) Let $S_{3}=\prec a, b \mid a^{3}=b^{2}=(a b)^{2}=1 \succ$ be the symmetric group of order 6 . One may directly show that $A B\left(S_{3}\right)=\left\{f \in C\left(S_{3}\right): f(1)=f(a)=f\left(a^{2}\right)\right.$, and $f(b)=$ $\left.f(a b)=f\left(a^{2} b\right)\right\}$. Of course, $B D\left(S_{3}\right)=\mathbb{C}$.

(iv) An inductive proof shows that a function $f \in W A P$ lies in $A B$ if and only if

$f$ (each finite product of elements of $S)=f$ (each re-ordering of it).

(v) Similar to what we have preceding to Proposition 3.3, using the Lawson's theorem, [8], one may show that for abelian semigroups $B D \cap W A P=S L=B D \cap A P$. Thus, for semilattices, $S L=A P$.

(vi) The equality $B D \cap M D=L Z$ can be easily demonstrated from the fact that all left simple bands are left zero semigroups. Similarly, $B D \cap S D=R Z$. Also, we trivially have $B D \cap G P=B D \cap S A P=\mathbb{C}$.

(vii) The invariant mean on the abelian semigroup $S^{A B}$ induces a unique invariant mean on $A B$, where the uniqueness is obtained from the fact that the $m$-admissible subalgebras of WAP cannot have more than one invariant mean (see [1, Cor. 2.3.28, Exereise 4.2.7]). A similar statement holds for $S L$ and $A B \cap G P$. But $B D$, in general, is not even left amenable. For example, for $S=\{a, b, c, d\}$ as in part (ii), let $f$ in $B D$ be such that $f(b) \neq f(c)$, then for each left invariant mean $m$ on $B D, f(b)=m\left(L_{b} f\right)=$ $m\left(L_{c} f\right)=f(c)$ and this contradicts the choice of $f$. 
(viii) It should be mentioned that $A B, S L, B D \cap D$, and $A B \cap G P$ are also admissible, i.e., they are invariant under $T_{\mu}$ for all $\mu$ in their duals [1, Cor. 4.2.7]. But we guess that $B D$ is not admissible in general. It would be desirable to investigate the inclusion $B D \subseteq W A P$.

(ix) Parallel to $B D$ and also $S L$ which are defined by right translates, we have the analogous spaces defined by left translates. It is a matter of fact that the left and right notations do not change the structure of $S L$ (see Proposition 3.3). A natural question that arises is whether they do not change $B D$. In our opinion, there is a close tie between the latter question and the inclusion $B D \subseteq W A P$. See (viii).

(x) It is obvious that the $S L$-compactification of the direct product of two semitopological semigroups is isomorphic (in the sense of [1, Sec. 5.2]) to the direct product of their $S L$-compactificatons. A similar fact holds for the $(A B \cap G P)$-compactification; (more generally, $(A B \cap G P)$-compactification, roughly speaking, passes through semidirect products. See [1, Lem. 5.2.3]).

ACKNOWLEDGEMENT. A research grant from IPM (Iran branch) is gratefully acknowledged.

\section{REFERENCES}

[1] J. F. Berglund, H. D. Junghenn, and P. Milnes, Analysis on semigroups: Function spaces, compactifications, representations, Canadian Mathematical Society Series of Monographs and Advanced Texts, John Wiley \& Sons, Inc., New York, 1989. MR 91b:43001. Zbl 727.22001.

[2] K. de Leeuw and I. Glicksberg, Applications of almost periodic compactifications, Acta Math. 105 (1961), 63-97. MR 24\#A1632. Zbl 104.05501.

[3] H. R. Ebrahimi Vishki and M. A. Pourabdollah, The universal nilpotent group compactification of a semigroup, Proc. Amer. Math. Soc. 125 (1997), no. 7, 2171-2174. MR 97i:43002. Zbl 970.31970.

[4] R. Ellis, Locally compact transformation groups, Duke Math. J. 24 (1957), 119-125. MR 19,561b. Zbl 079.16602.

[5] E. Hewitt and K. A. Ross, Abstract harmonic analysis. Vol. I: Structure of topological groups, Integration theory, group representations, Die Grundlehren der mathematischen Wissenschaften, vol. 115, Academic Press, Inc., Publishers and Springer-Verlag, New York and Berlin Gottingen Heidelberg, 1963. MR 28\#158. Zbl 115.10603.

[6] H. D. Junghenn, Distal compactifications of semigroups, Trans. Amer. Math. Soc. 274 (1982), no. 1, 379-397. MR 84h:43017. Zbl 514.22003.

[7] H. D. Junghenn and R. D. Pandian, Existence and structure theorems for semigroup compactifications, Semigroup Forum 28 (1984), no. 1-3, 109-122. MR 85c:22005. Zbl 531.22004.

[8] J. D. Lawson, Joint continuity in semitopological semigroups, Illinois J. Math. 18 (1974), 275-285. MR 49 454. Zbl 278.22002.

[9] R. D. Pandian, Semigroup compactifications by generalized distal functions and a fixed point theorem, Internat. J. Math. Math. Sci. 14 (1991), no. 2, 253-260. MR 92j:54057. Zbl 739.43009 .

[10] _ Quasiminimal distal function space and its semigroup compactification, Internat. J. Math. Math. Sci. 18 (1995), no. 3, 497-500. MR 96e:22007. Zbl 840.43013.

ViSHKI AND POURABDOLLAH: FACULTY OF MATHEMATICS, UNIVERSITY OF MASHHAD, P.O. BOX 91775-1159, MASHHAD, IRAN 


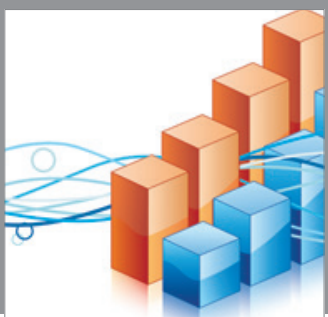

Advances in

Operations Research

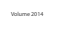

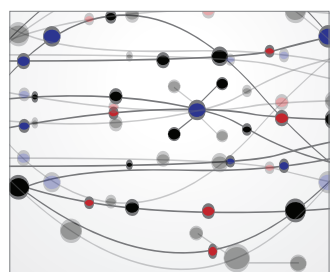

\section{The Scientific} World Journal
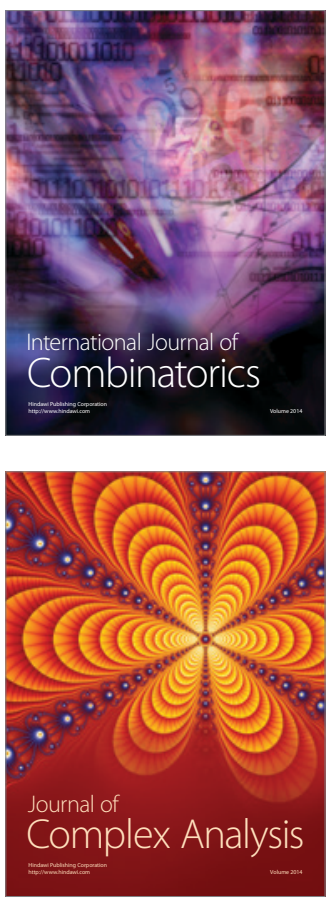

International Journal of

Mathematics and

Mathematical

Sciences
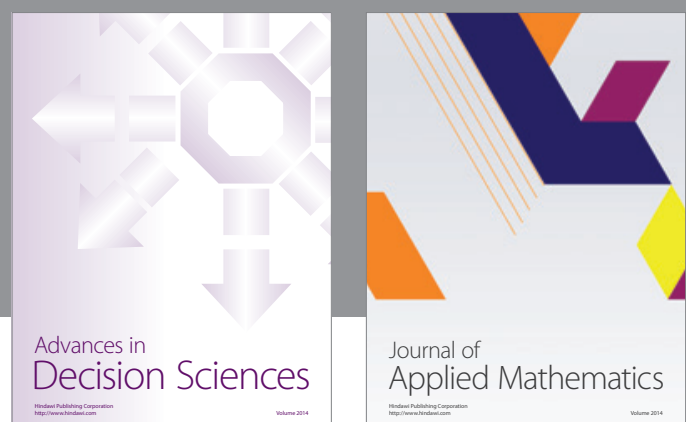

Journal of

Applied Mathematics
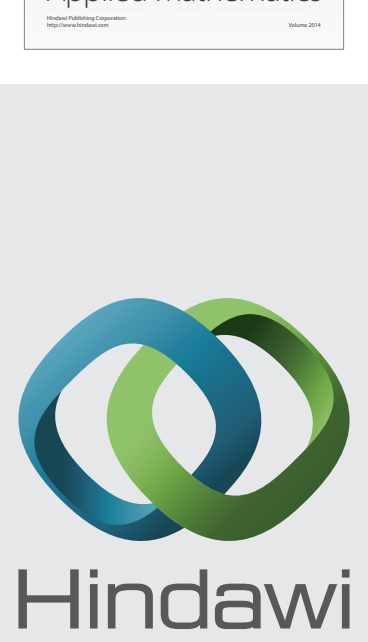

Submit your manuscripts at http://www.hindawi.com
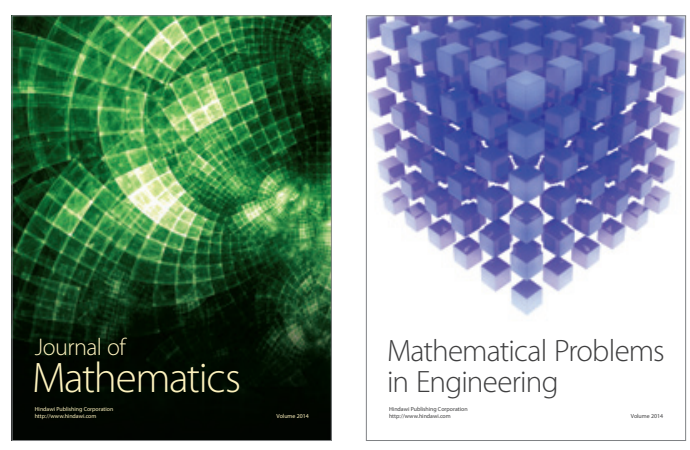

Mathematical Problems in Engineering
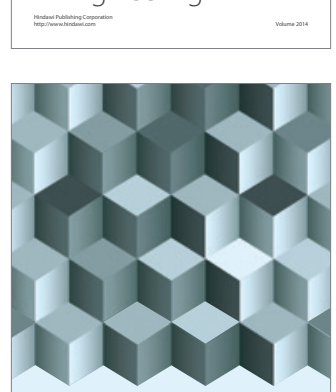

Journal of

Function Spaces
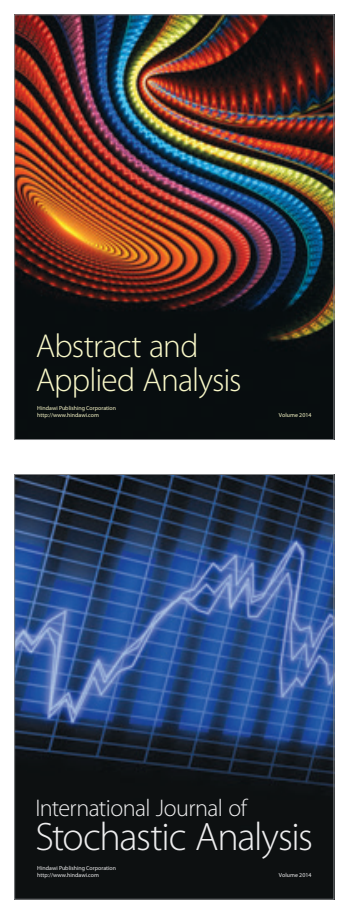

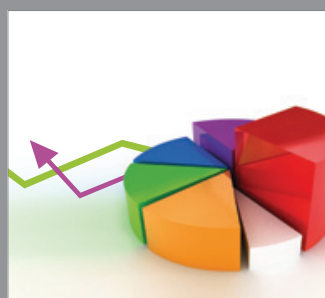

ournal of

Probability and Statistics

Promensencen
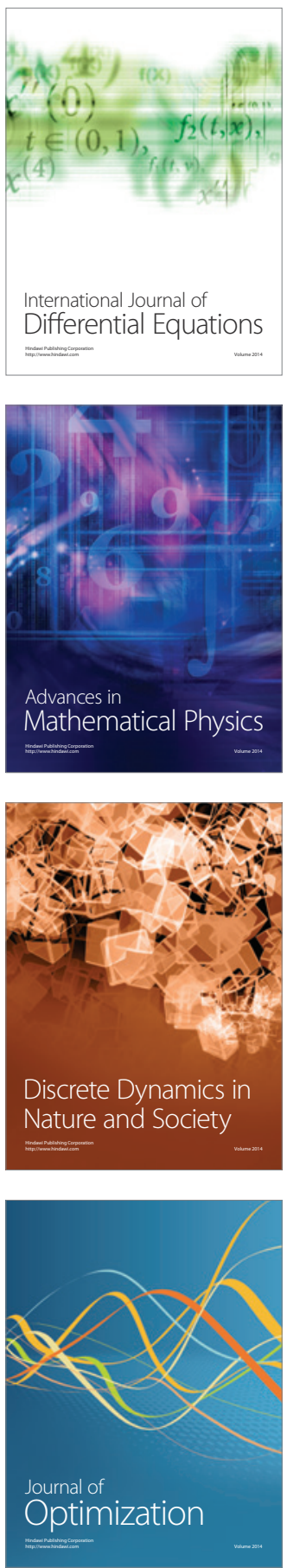